\title{
Public Willingness to Undergo Presymptomatic Genetic Testing for Alzheimer's Disease
}

\author{
Mohammed H. Alanazy (D), Khalid A. Alghsoon, Abdulaziz F. Alkhodairi, \\ Faisal K. Binkhonain, Turkey Naaser Alsehli, Feras F. Altukhaim, Ibrahim M. Alkhodair, \\ and Taim Muayqil
}

\begin{abstract}
Division of Neurology, Department of Internal Medicine, King Saud University Medical City and College of Medicine, King Saud University, Riyadh, Saudi Arabia
\end{abstract}

Correspondence should be addressed to Mohammed H. Alanazy; dranazy@gmail.com

Received 24 November 2018; Revised 20 January 2019; Accepted 3 February 2019; Published 3 March 2019

Academic Editor: Changiz Geula

\begin{abstract}
Copyright (C) 2019 Mohammed H. Alanazy et al. This is an open access article distributed under the Creative Commons Attribution License, which permits unrestricted use, distribution, and reproduction in any medium, provided the original work is properly cited.
\end{abstract}

\begin{abstract}
Presymptomatic genetic testing (PGT) for Alzheimer's disease (AD) is available for at-risk individuals. This study aimed to investigate the public perception of PGT in Saudi Arabia and determine variables that might influence the decision to undergo PGT. A questionnaire link was posted on Twitter by the Saudi Alzheimer's Disease Association and was made publicly available on social media networks. A total of 2935 people participated, of which $59.9 \%$ were willing to undergo PGT. Of these, $26.8 \%$ reported having a family history of $\mathrm{AD}$, and $0.24 \%$ had two family members with early onset $\mathrm{AD}$. The reasons cited for willingness to undergo PGT included the following: to adopt a healthier lifestyle, to ensure appropriate family and financial planning, to seek early treatment, and to relieve anxiety. In multiple logistic regression analysis, willingness to undergo PGT was negatively associated with having a self-reported family history of dementia (OR 0.81, 95\% CI 0.68-0.96) and was positively associated with marital status (OR 1.39 , 95\% CI 1.13-1.70). In conclusion, PGT for AD seems to be well accepted in this large Saudi cohort. The reasons cited are similar to those reported elsewhere in the literature.
\end{abstract}

\section{Introduction}

Alzheimer's disease (AD), the most common form of dementia, presents as a progressive decline in cognition, behavior, and functional abilities and develops as a consequence of aggregation of $\beta$-amyloid and hyperphosphorylated tau in vulnerable brain regions [1]. The cognitive domains affected by $\mathrm{AD}$ mostly involve short-term memory, visuospatial function, executive function, and language. The diagnosis of $\mathrm{AD}$ is typically made on the basis of clinical and cognitive assessment; however, recent advances in neuroimaging, cerebrospinal fluid biomarkers, and genetic testing can facilitate early and presymptomatic diagnosis. The predominant form of the disease is late-onset $\mathrm{AD}$ (LOAD), which usually manifests at or after 65 years of age. Early onset AD (EOAD) accounts for less than $5 \%$ of cases and has an age of onset below 65 years. A fully penetrant autosomal dominant form of EOAD is caused by mutations in the amyloid precursor protein $(A P P)$ and presenilin-1 (PSEN1) genes, whereas the penetrance of mutations in presenilin-2 (PSEN2) gene is 95\% [2]. In contrast, individuals with one copy of the apolipoprotein $\mathrm{E}(A P O E) * E 4$ allele have a three- to fourfold increase in the risk of developing $\mathrm{AD}$, particularly at an older age $[2,3]$. The risk is higher in individuals who have two copies of the $A P O E * E 4$ allele $[2,3]$. However, $A P O E$ is not a deterministic gene but rather considered a susceptibility gene, and the risk conferred by the $E 4$ allele differs based on age, sex, and ethnicity $[2,3]$. Due to its limited clinical utility, genetic testing for $A P O E$ is not recommended [2].

Identification of dominantly inherited genes and the revolution in genomic technology has made genetic testing readily available for diagnostic purposes in symptomatic individuals and for predictive purposes in asymptomatic individuals with a family history of $\mathrm{AD}$ [2]. Identifying $\mathrm{AD}$ mutation carriers in the presymptomatic stage facilitates enrollment in preclinical treatment trials, with the ultimate 
goal of initiating therapy before the manifestation of irreversible brain damage [4]. Presymptomatic genetic testing (PGT) should always be preceded by genetic counseling and is preferably performed on the basis of a positive result in an affected family member [2]. Caselli et al. reported that an online web-based survey revealed that $80.8 \%$ of respondents were willing to undergo PGT for AD [5]. The population of that study was limited to participants who were interested in $\mathrm{AD}$ prevention research and had registered with an $\mathrm{AD}$ prevention registry website, which likely explains the high willingness to undergo PGT. In addition, public perception of PGT may vary across locations and may be influenced by cultural and religious beliefs of a society. Thus, this study sought to assess public perception of PGT for AD in Saudi Arabia and explore factors that might influence willingness to participate in genetic testing.

\section{Methods}

This study was approved by the institutional review board of King Saud University. A questionnaire link was posted on Twitter by the Saudi Alzheimer's Disease Association (approximately 38,000 followers) and was made publicly available on social media networks during the period from April to August 2018. Participants were encouraged to forward the survey link to their social networks, resulting in a snowball sample. To prevent duplicate responses, the survey was programmed to allow only one response per IP address (using surveymonkey.com).

The first draft of the questionnaire was developed by all authors in Arabic, based on literature review and taking into consideration the general culture of the Saudi society. The questionnaire was subsequently reviewed by 2 neurologists for completeness and comprehension. A group of randomly selected healthy volunteers of different ages and educational levels were asked to assess the coherency and readability of the questionnaire. In total, 3 successive versions of the questionnaire were tested in 20 healthy volunteers before it was finalized. The questionnaire was structured into the following sections: (1) an introductory statement briefly explaining the disease with emphasis on the current absence of disease-modifying therapy, (2) informed consent where participants consented if they select "agree to participate" button, (3) participant demographics, (4) questions regarding family history of $\mathrm{AD}$ or dementia, (5) a question related to the participant's experience of being a caregiver for a patient with $\mathrm{AD},(6)$ questions regarding the participant's willingness to undergo PGT for AD, and (7) the participant's reasons for undergoing or rejecting PGT (maximum of 3 ). The questions regarding PGT were structured to be self-explanatory, e.g., for autosomal dominant $\mathrm{AD}$ genes, "if the gene being tested is inherited in a dominant manner and its presence means you will certainly develop $\mathrm{AD}$ at a young age (generally before 50 years), would you agree to do this test?" and for $A P O E$ "if the gene being tested increases your risk of developing $\mathrm{AD}$ at an older age (generally after 65 years) but without certainty (as there are people who have this gene and do not develop AD), would you agree to do this test?"
TABLE 1: Characteristics of the study population (total number of participants $=2935$ ).

\begin{tabular}{lc}
\hline Participants & Number $(\%)$ \\
\hline Sex & \\
Female & $1614(55.0)$ \\
Male & $1321(45.0)$ \\
\hline Age & \\
18-30 years & $1158(39.5)$ \\
31-40 years & $749(25.5)$ \\
41-50 years & $597(20.3)$ \\
51-60 years & $336(11.4)$ \\
$>60$ years & $95(3.2)$ \\
\hline Marital status & \\
Married & $1757(59.9)$ \\
Not married (single, divorced, widowed) & $1178(40.1)$ \\
Nationality & \\
Saudi & $2780(94.7)$ \\
Non-Saudi & $155(5.3)$ \\
\hline City of residence & $1949(66.4)$ \\
In Riyadh & $986(33.6)$ \\
Out of Riyadh & $13(0.4)$ \\
\hline Highest level of education & $32(1.1)$ \\
No schooling & $67(2.3)$ \\
Primary school & $615(21.0)$ \\
Secondary school & $286(9.7)$ \\
High school & $117(4.0)$ \\
Diploma & \\
Bachelor's degree & \\
Master's degree & \\
PhD & \\
\hline & \\
& \\
\hline
\end{tabular}

\section{Analysis}

Demographic and categorical data were reported as numbers and percentages. Multiple logistic regression analysis was employed to determine characteristics associated with a decision to undergo or reject PGT for $\mathrm{AD}$. Independent variables included age, sex, marital status, highest level of education, having a first- or second-degree relative with dementia, and having been a caregiver for a patient with dementia. An odds ratio (OR) and 95\% confidence interval (CI) were calculated for each independent variable. A twosided $\mathrm{P}$ value $<0.05$ was considered significant. Data analysis was conducted using statistical software SPSS, version 23 (IBM, Armonk, NY).

\section{Results}

Of 3060 participants, 2935 (55\% women, 45\% men) returned complete questionnaires. Incomplete questionnaires were not included in the analysis. Table 1 shows the demographics and characteristics of the participants. The majority of participants were $18-30$ years old (39.5\%), married (59.9\%), living in Riyadh (66.4\%), of Saudi nationality (94.7\%), and 
TABLE 2: Participants' report of family history of Alzheimer's disease, dementia, and age of onset before 50 years (total number of participants =2935).

\begin{tabular}{|c|c|c|}
\hline Participants' report & Number $(\%)$ & $\begin{array}{c}\text { Onset before } 50 \text { years of } \\
\text { age; number }(\%)\end{array}$ \\
\hline First-degree relative with $\mathrm{AD}$ & $363(12.4)$ & $24(0.82)$ \\
\hline Second-degree relative with $\mathrm{AD}$ & $584(19.9)$ & $19(0.53)$ \\
\hline Both first- and second-degree relatives with $\mathrm{AD}$ & $161(5.5)$ & $7(0.24)$ \\
\hline First- or second-degree relative with $\mathrm{AD}$ & $786(26.8)$ & NA \\
\hline First-degree relative with dementia & $536(18.3)$ & $33(1.12)$ \\
\hline Second-degree relative with dementia & $870(29.6)$ & $28(0.95)$ \\
\hline Both first- and second-degree relatives with dementia & $273(9.3)$ & $11(0.37)$ \\
\hline First- or second-degree relatives with dementia & $1133(38.6)$ & NA \\
\hline Being a caregiver for a patient with $\mathrm{AD}$ & $526(17.9)$ & NA \\
\hline
\end{tabular}

TABle 3: Participants' perception about presymptomatic genetic testing for Alzheimer's disease (total number of participants =2935).

\begin{tabular}{lr}
\hline Participants' perception about presymptomatic genetic testing for autosomal dominant AD genes, APOE, or both. & Number (\%) \\
\hline Accepting either genetic tests & $1758(59.9)$ \\
Accepting both genetic tests & $1325(45.1)$ \\
Accepting autosomal dominant AD genetic test & $1560(53.2)$ \\
Accepting APOE gene test & $1523(51.9)$ \\
Rejecting both tests & $1177(40.1)$ \\
\hline
\end{tabular}

had a bachelor's degree as their highest level of education $(52.7 \%)$.

Table 2 shows participants' family histories of $\mathrm{AD}$ and dementia. Family history of a first- or second-degree relative with AD was reported by 786 participants (26.8\%). Family history of having both a first- and second-degree relative with $\mathrm{AD}$ was reported by 161 participants $(5.5 \%), 7$ (0.24\%) of whom reported that the onset was before 50 years of age. Of all participants, $526(17.9 \%)$ reported having been a caregiver for a patient with $\mathrm{AD}$.

Regarding participants' willingness to have PGT for AD, $59.9 \%$ were willing to undergo PGT for either the autosomal dominant genes or the APOE, whereas $40.1 \%$ rejected both tests (Table 3). The most frequently selected reasons for accepting PGT (1758 participants; Table 4) were "to adopt a healthy lifestyle" (52.1\%), "to make plans for future and family planning" (41.5\%), "possibility of finding a cure for the disease in the future" (40.0\%), and "to relieve anxiety" (37.8\%). A minority (4.7\%) provided descriptive reasons that were mostly related to "having forgetfulness" and "a desire to participate in research." Of those who expressed willingness to undergo PGT, 76.5\% indicated that their acceptance would be contingent upon the test being offered free. The most frequently selected reasons for rejecting PGT (1177 participants, Table 5) were "worried about developing a mental illness after knowing the results" (32.5\%), "fear of the result of genetic testing" (27.7\%), and "absence of cure" (22.9\%). A minority $(5.8 \%)$ provided descriptive reasons that were mostly related to spiritual faith and an absence of family history of dementia.

In the logistic regression analysis, only two factors were found to contribute significantly to participants' willingness to undergo PGT. Participants with family history of a first- or second-degree relative with dementia were less likely to accept PGT ( $\mathrm{OR}=0.81,95 \% \mathrm{CI}=0.68-0.96$, and $\mathrm{P}=0.02)$, and married participants were more likely to accept PGT ( $\mathrm{OR}=1.39,95 \% \mathrm{CI}=1.13-1.70$, and $\mathrm{P}=0.002)$.

\section{Discussion}

This is the first study on public perception of PGT for AD in Saudi Arabia and one of the few studies undertaken to date worldwide. In this study, more than half of participants showed willingness to undergo PGT for AD. Married individuals are more likely to accept PGT testing, while those with a family history are less likely to do so. A conjectural explanation is that married individuals may want to know their risk of developing $\mathrm{AD}$ to help them with family planning (e.g., having children). In contrast, individuals who have a relative with dementia may not want to know the risk to avoid the emotional and psychological consequences of knowing that their health will deteriorate similarly to that of their loved ones. In contrast, Caselli et al. reported that male sex and number of affected relatives are associated with a higher likelihood of agreeing to PGT and higher education is associated with a lower likelihood [5]. This discrepancy between the two studies may be influenced by the differences in religion, culture, beliefs, and values of the studied populations. In Islam, premarital genetic testing is considered an acceptable procedure [6]. The high frequency of consanguineous marriage in Saudi Arabia is one of the cultural factors that contributes to the high prevalence of inherited hemoglobinopathies [7] and, conceivably, to the increased risk of other autosomal recessive diseases (AR). Therefore, the premarital screening and genetic counselling 
TABLE 4: Reasons for accepting presymptomatic genetic testing for Alzheimer's disease (total responses 1758).

\begin{tabular}{lr}
\hline Reasons & Number of responses (\%) \\
\hline To adopt a healthy lifestyle & $916(52.1 \%)$ \\
To make plans for future and family planning & $730(41.5 \%)$ \\
The possibility of finding a cure for Alzheimer's disease in the future & $703(40.0 \%)$ \\
To relieve anxiety & $664(37.8 \%)$ \\
To make plans regarding my job and financial planning & $396(22.5 \%)$ \\
I know a person with Alzheimer disease & $221(12.6 \%)$ \\
Other & $82(4.7 \%)$ \\
\hline
\end{tabular}

TABLE 5: Reasons for rejecting presymptomatic genetic testing for Alzheimer's disease (total responses 1177).

\begin{tabular}{lc}
\hline Reasons & Number of responses (\%) \\
\hline Worried about developing a mental illness after knowing the result & $383(32.5 \%)$ \\
Fear of the result of genetic testing & $326(27.7 \%)$ \\
No cure for Alzheimer disease currently & $269(22.9 \%)$ \\
Worried that the result will affect my employment & $116(9.9 \%)$ \\
I do not trust genetic tests & $104(8.8 \%)$ \\
Afraid from financial consequences of the medical care & $33(2.8 \%)$ \\
Worried about discrimination by insurance companies & $31(2.6 \%)$ \\
Other & $68(5.8 \%)$ \\
\hline
\end{tabular}

program (PMSGC) has been established and made mandatory to reduce the burden of AR diseases, specifically sicklecell disease and $\beta$-thalassemia [7]. The PMSGC has been successful in reducing the prevalence of $\beta$-thalassemia [7]. Yet, $48 \%$ of at-risk marriages were completed in 2009 , indicating that the cultural challenges were not fully addressed by the genetic counselors [7].

Secondly, the discrepancy between our study and the study by Caselli et al. may be related to differences in the participant samples. In our study, the survey link was available to all adults in the community, whereas in that study by Caselli et al., it was confined to those registered with an $\mathrm{AD}$ prevention registry website [5]. Other studies of at-risk individuals reported that $54 \%$ accepted PGT for neurodegenerative diseases [8], and 58\% accepted PGT for Huntington's disease and autosomal dominant spinocerebellar ataxia [9]. Notably, the number of eligible individuals seeking PGT typically drops following genetic counseling, reflecting the complexity of the decision-making process $[10,11]$.

Common reasons cited for unwillingness to undergo PGT in this and previous studies include fear of the consequences of the results [5], absence of curative therapy [8], and fear of the psychological impact [5]. Despite the current absence of disease modifying or preventative therapy, most at-risk individuals that are tested for hereditary ataxias and neuromuscular disorders find PGT beneficial and cope well regardless of the results. However, the impact of PGT results on anxiety and depression is unpredictable [12]. Generally, even individuals with normal test results experience conflicting emotions of happiness, guilt, relief, fear, and anger [13]. While psychological support is required for all PGT seekers [14], some may experience severe distress after receiving a result [15] and require intensive support from health professionals experienced in counseling.
Slooter et al. [16] reported that $25 \%$ of the general population aged 55 years and older have a first-degree relative with dementia. Our results demonstrate a lower rate (18.3\%), perhaps because the majority of our participants were younger (Tables 1 and 2). However, in this study, a notable proportion of the participants reported having a first- or second-degree relative with $\mathrm{AD}(26.8 \%)$ and dementia (38.6\%). While this study was not designed to assess the prevalence of dementia in Saudi Arabia, and reported diagnoses were not investigated, our data may reflect a high prevalence rate of $\mathrm{AD}$ and dementia that is not unexpected in view of the population growth and aging, the high illiteracy rate among people older than 65 years [17], and the high prevalence of cardiovascular risk factors [18]. Using a validated Arabic version of the Montreal cognitive assessment test (MOCA), a previous study reported a prevalence of dementia of $26 \%$ among Saudis attending primary care clinics, which decreased to $6.4 \%$ after using an education-adjusted MOCA score [19]. However, those data were obtained by convenience sampling; more representative samples would be required to better estimate the population prevalence of $\mathrm{AD}$ and dementia in Saudi Arabia.

Autosomal dominant $\mathrm{AD}$ has a younger age of onset compared with sporadic LOAD, but variations have been observed within families and various mutation types [20]. We decided to use the age of 50 years (rather than 60 years) to define young onset $\mathrm{AD}$. This was supported by a meta-analysis of symptom onset in autosomal dominant AD that estimated a mean age of onset to be 46.2 years [20]. In addition, we took into consideration the Saudi culture, whereby people cite their age using the Hijri calendar in which a person's age is older by approximately one year for every 30 years in the Gregorian calendar. Most older people may not know their exact date of birth either because they 
were born in a rural-Bedouin society or born prior to the development of stringent governmental birth record keeping. For these reasons, older Saudi Arabians may not report their age, or the age of their elderly relatives, accurately. Therefore, we decided to choose a more conservative age limit when identifying EOAD.

EOAD has been estimated to comprise $1-5 \%$ of all $\mathrm{AD}$ cases [21], and autosomal dominant AD accounts for 1-2\% [4]. Among all participants in this study, 5.5\% reported having both a first- and second-degree relative with $\mathrm{AD}$, and $0.24 \%$ reported that the onset was before the age of 50 years. Notably, these data are based on information about diagnosis and age of onset as perceived by the participants, rather than as ascertained by a physician. Nonetheless, it is reasonable to consider those participants who reported having 2 family members with EOAD to have a potential risk for $\mathrm{AD}$.

In addition to the discussed limitations above, although the questionnaire link was open to all adults from the society, sampling bias cannot be excluded. It is possible that there was a bias in participation from people who are interested in or have a family history of AD. Furthermore, input from people who do not have access to internet and social media, especially those older than 50 years, was limited due to our data collection method.

In conclusion, willingness to undergo PGT was expressed by approximately $60 \%$ of our study population. Significant associations were observed between participants' willingness to undergo PGT and their marital status as well as selfreported family history of dementia. In addition, the study explored variables that might influence the decision to undergo PGT. Pending further prospective studies, these findings may be helpful to physicians to develop a general framework when counseling relatives of patients with $\mathrm{AD}$.

\section{Data Availability}

The data used to support the findings of this study are available from the corresponding author upon request.

\section{Conflicts of Interest}

The authors declare that there are no conflicts of interest regarding the publication of this paper.

\section{Acknowledgments}

This work was supported by the College of Medicine Research Center, Deanship of scientific Research, King Saud University, Riyadh, Saudi Arabia. The authors thank the Saudi Alzheimer's Disease Association for their support in distributing the questionnaire.

\section{References}

[1] Alzheimer's Association, "Alzheimer's disease facts and figures," Alzheimer Dement, vol. 12, pp. 459-509, 2016.

[2] J. S. Goldman, S. E. Hahn, J. W. Catania, S. LaRusse-Eckert, M. B. Butson, and M. Rumbaugh, "Genetic counseling and testing for Alzheimer disease: Joint practice guidelines of the American College of Medical Genetics and the National Society of genetic counselors," Genetics in Medicine, vol. 13, pp. 597-605, 2011.

[3] J. M. Ringman and G. Coppola, "New genes and new insights from old genes: Update on Alzheimer disease," Continuum: Lifelong Learning in Neurology, vol. 19, no. 2, pp. 358-371, 2013.

[4] P. N. Tariot, F. Lopera, J. B. Langbaum et al., "The alzheimer's prevention initiative autosomal-dominant Alzheimer's disease trial: a study of crenezumab versus placebo in preclinical PSEN1 E280A mutation carriers to evaluate efficacy and safety in the treatment of autosomal-dominant Alzheimer's disease, including a placebo-treated noncarrier cohort," Alzheimer's and Dementia: Translational Research and Clinical Interventions, vol. 4, pp. 150-160, 2018.

[5] R. J. Caselli, J. Langbaum, G. E. Marchant et al., "Public perceptions of presymptomatic testing for Alzheimer disease," Mayo Clinic Proceedings, vol. 89, no. 10, pp. 1389-1396, 2014.

[6] M. A. F. El-Hazmi, "Ethics of genetic counseling - Basic concepts and relevance to Islamic communities," Annals of Saudi Medicine, vol. 24, no. 2, pp. 84-92, 2004.

[7] Z. A. Memish and M. Y. Saeedi, "Six-year outcome of the national premarital screening and genetic counseling program for sickle cell disease and -thalassemia in Saudi Arabia," Annals of Saudi Medicine, vol. 31, no. 3, pp. 229-235, 2011.

[8] C. S. M. Rodrigues, V. Z. De Oliveira, G. Camargo et al., "Presymptomatic testing for neurogenetic diseases in Brazil: Assessing who seeks and who follows through with testing," Journal of Genetic Counseling, vol. 21, no. 1, pp. 101-112, 2012.

[9] C. Goizet, G. Lesca, and A. Dürr, "Presymptomatic testing in Huntington's disease and autosomal dominant cerebellar ataxias," Neurology, vol. 59, no. 9, pp. 1330-1336, 2002.

[10] S. Wedderburn, P. K. Panegyres, S. Andrew et al., "Predictive gene testing for Huntington disease and other neurodegenerative disorders," Internal Medicine Journal, vol. 43, no. 12, pp. 1272-1279, 2013.

[11] S. Reyes, A. Kurtz, D. Hervé, E. Tournier-Lasserve, and H. Chabriat, "Presymptomatic genetic testing in CADASIL," Journal of Neurology, vol. 259, no. 10, pp. 2131-2136, 2012.

[12] C. O. Smith, H. P. Lipe, and T. D. Bird, "Impact of presymptomatic genetic testing for hereditary ataxia and neuromuscular disorders," JAMA Neurology, vol. 61, no. 6, pp. 875-880, 2004.

[13] L. Godino, D. Turchetti, L. Jackson, C. Hennessy, and H. Skirton, "Impact of presymptomatic genetic testing on young adults: A systematic review," European Journal of Human Genetics, vol. 24, no. 4, pp. 496-503, 2016.

[14] M. Gargiulo, S. Lejeune, M.-L. Tanguy et al., "Long-term outcome of presymptomatic testing in Huntington disease," European Journal of Human Genetics, vol. 17, no. 2, pp. 165-171, 2009.

[15] R. J. Tassicker, P. K. Marshall, T. A. Liebeck, M. A. Keville, B. M. Singaram, and F. H. Richards, "Predictive and prenatal testing for Huntington disease in Australia: Results and challenges encountered during a 10-year period (1994-2003)," Clinical Genetics, vol. 70, no. 6, pp. 480-489, 2006.

[16] A. J. C. Slooter, M. Cruts, S. Kalmijn et al., "Risk estimates of dementia by apolipoprotein E genotypes from a populationbased incidence study: the Rotterdam study," JAMA Neurology, vol. 55, no. 7, pp. 964-968, 1998.

[17] "The General Authority for Statistics. Demographic Survey 2016," https://www.stats.gov.sa/sites/default/files/en-demographic-research-2016_2.pdf. 
[18] A. M. Ahmed, A. Hersi, W. Mashhoud et al., "Cardiovascular risk factors burden in Saudi Arabia: The Africa Middle East Cardiovascular Epidemiological (ACE) study Cardiovascular risk factors burden in Saudi Arabia," Journal of the Saudi Heart Association, vol. 29, no. 4, pp. 235-243, 2017.

[19] M. Alkhunizan, A. Alkhenizan, and L. Basudan, "Prevalence of mild cognitive impairment and dementia in Saudi Arabia: a community-based study," Dementia and Geriatric Cognitive Disorders Extra, vol. 8, no. 1, pp. 98-103, 2018.

[20] D. C. Ryman, N. Acosta-Baena, P. S. Aisen, T. Bird, A. Danek, N. C. Fox et al., "Symptom onset in autosomal dominant Alzheimer disease," Neurology, vol. 83, pp. 253-260, 2014.

[21] C. Reitz, C. Brayne, and R. Mayeux, "Epidemiology of Alzheimer disease," Nature Reviews Neurology, vol. 7, no. 3, pp. 137-152, 2011. 


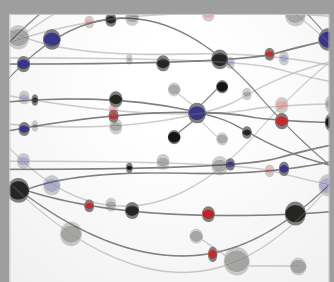

The Scientific World Journal
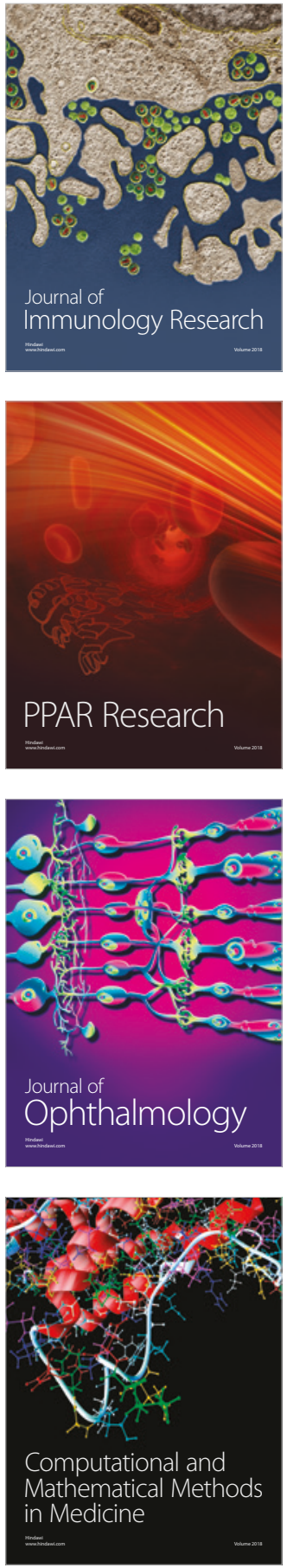

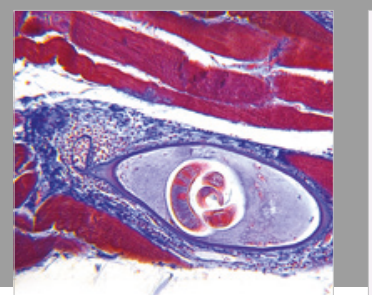

Gastroenterology Research and Practice

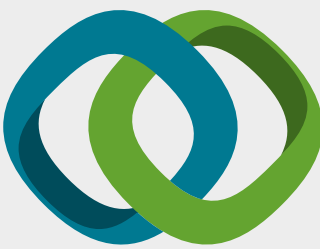

\section{Hindawi}

Submit your manuscripts at

www.hindawi.com
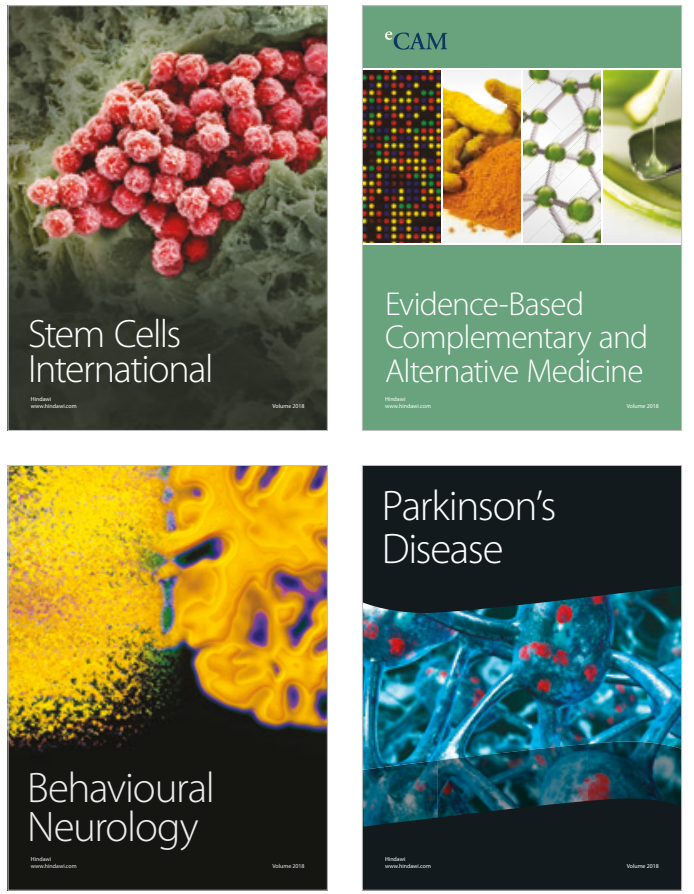

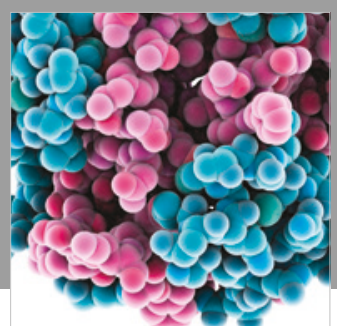

ournal of

Diabetes Research

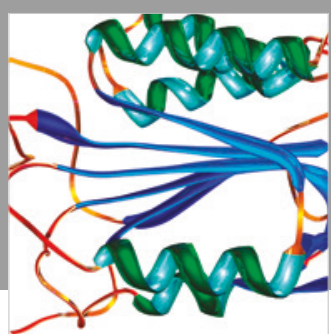

Disease Markers
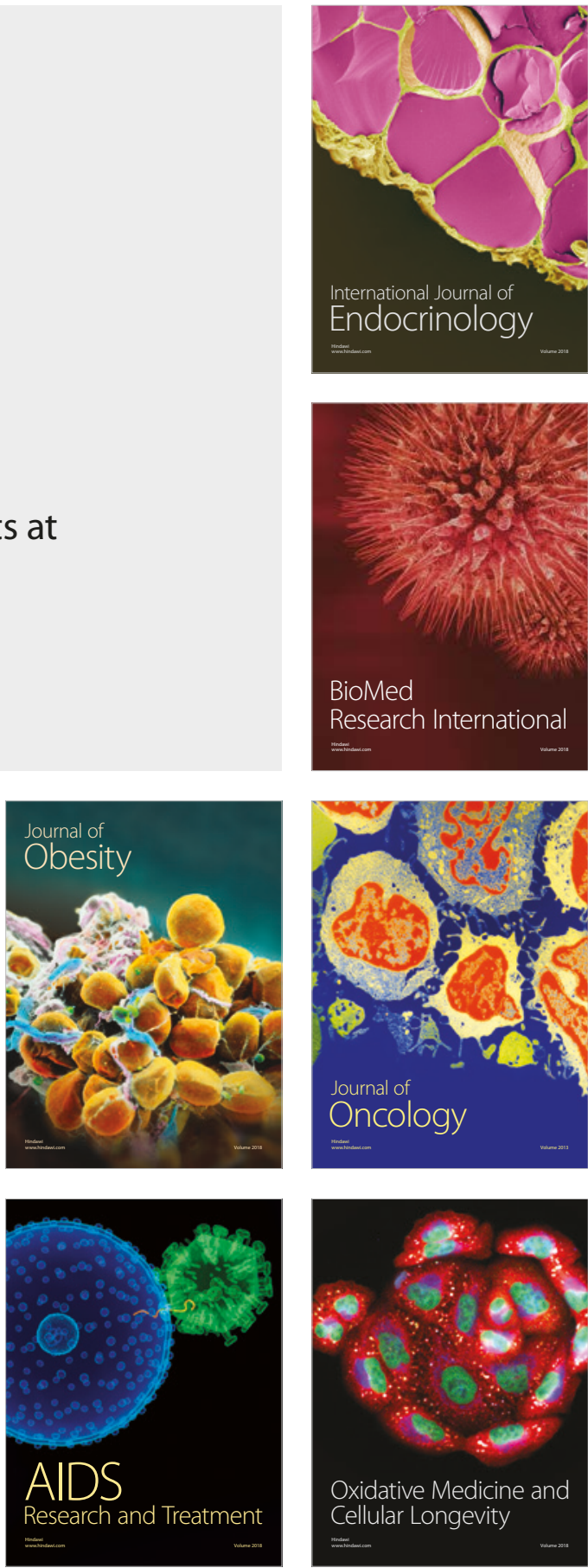